\title{
\begin{tabular}{l|l} 
Mitraries & DSpace@MIT
\end{tabular}
}

\author{
MIT Open Access Articles
}

\author{
Towards More Efficient, Greener \\ Syntheses through Flow Chemistry
}

The MIT Faculty has made this article openly available. Please share how this access benefits you. Your story matters.

Citation: Lummiss, Justin A.M. et al. “Towards More Efficient, Greener Syntheses through Flow Chemistry." The Chemical Record 17, 7 (February 2017): 667-680 (c) 2017 Wiley-Verlag

As Published: https://doi.org/10.1002/tcr.201600139

Publisher: Wiley Blackwell

Persistent URL: http://hdl.handle.net/1721.1/114543

Version: Author's final manuscript: final author's manuscript post peer review, without publisher's formatting or copy editing

Terms of use: Creative Commons Attribution-Noncommercial-Share Alike 


\section{Towards More Efficient, Greener Syntheses Through Flow Chemistry}

\author{
Justin A.M. Lummiss, ${ }^{[a]}$ Peter D. Morse, ${ }^{[a]}$ Rachel \\ L. Beingessner, ${ }^{[a]}$ and Timothy F. Jamison*[a]
}

\begin{abstract}
Technological advances have an important role in the design of greener synthetic processes. In this Personal Account, we describe a wide range of thermal, photochemical, catalytic, and biphasic chemical transformations examined by our group. Each of these demonstrate how the merits of a continuous flow synthesis platform can align with some of the goals put forth by the Twelve Principles of Green Chemistry. In particular, we illustrate the potential for improved reaction efficiency in terms of atom economy, product yield and reaction rates, the ability to design synthetic process with chemical and solvent waste reduction in mind as well as highlight the benefits of the realtime monitoring capabilities in flow for highly controlled synthetic output.
\end{abstract}

\section{Introduction}

First outlined by Anastas and Warner in $1998,{ }^{[1]}$ the Twelve Principles of Green Chemistry provide a valuable framework for evaluating the efficiency and sustainability of a given chemical transformation or process. ${ }^{[2]}$ On the molecular level, these principles call for the design of reactions that are more atom economical, minimize the use of hazardous reagents, and utilize renewable feedstocks. The design of reaction protocols with energy efficiency in mind, and employing real-time reaction analysis in order to minimize waste generation are also among the recommendations. Technological advances, such as continuous flow synthesis, have an important role to play in advancing these goals. ${ }^{[3]}$

Continuous flow reactions on the meso- to macro-scale level are typically carried out within commercially available small diameter tubing (inner diameter 0.01-0.080") fabricated from either polymer (e.g. perfluoroalkoxy alkanes, Tefzel, polyetheretherketone) or metal (e.g. stainless steel, copper) materials. ${ }^{[4]}$ A range of Swagelok and HPLC fittings enable the relatively facile assembly of bespoke flow set-ups using some or all of the components showcased in Figure $1 a$ and $b$.

[a] Dr. J. A. M. Lummiss, Dr. P. D. Morse, Dr. R. L. Beingessner, Professor T. F. Jamison Department of Chemistry Massachusetts Institute of Technology 77 Massachusetts Avenue, Cambridge, MA, 02139, USA E-mail: tfj@mit.edu
Alternatively, entire flow systems with specialized attachments can be purchased from various commercial suppliers. ${ }^{[\mathrm{b}]}$

(a)

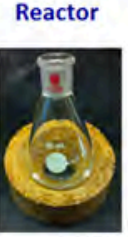

Mixing

Separating

Pressurizing

Batch
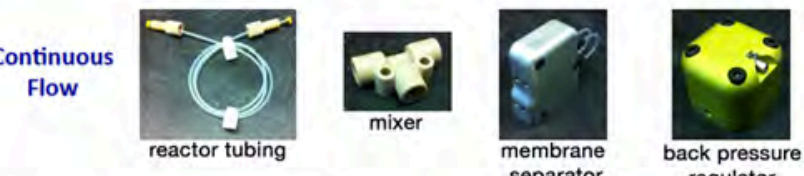

(b)
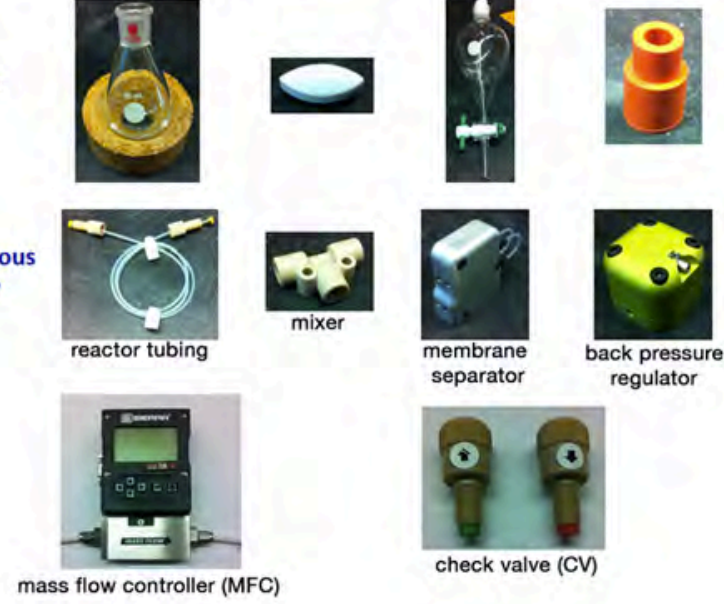

regulator

Figure 1. (a) Comparison of the major unit operations in batch relative to flow. (b) Additional tools utilized in flow reactions including a (left) mass flow controller (MFC), which is used to regulate gas flow and (right) check valves (CV), which are used to prevent back flow.

There are several benefits of flow chemistry compared to traditional batch synthesis. ${ }^{[3 e, 5]}$ For example, the high surface area to volume ratio of a flow reactor enables superior mixing of biphasic reactions, such as those between gases and liquids, which can significantly improve product yields. ${ }^{[5 b]}$ This same attribute also permits precise temperature control, which can increase reaction efficiency (e.g. reaction rate, product yield) while minimizing energy consumption. ${ }^{[3 \mathrm{~d}]}$ Photochemical transformations can similarly benefit from improved yields, decreased reaction time-scales, and reduced catalyst loadings, due to the highly efficient irradiation that results from having a short path length. ${ }^{[5 d, 6]}$

Another distinguishing feature of continuous flow is that the amount of product generated is determined by the length of time the entire flow regime is operated, given defined flow rates and reactor volumes. This is in contrast to batch, where the maximum quantity of product produced per reaction, is predetermined by the quantity of starting material. It is this very nature of continuous production that enables small volume flow reactors to produce comparably large quantities of product. The reduced reactor volume in flow also enables more efficient engineering controls and also inherently reduces safety risks by avoiding large accumulations of potentially hazardous intermediates at any given point in time. 
A major research focus of our group lies in leveraging continuous flow platforms to improve reaction outcomes and enable transformations that are difficult, or simply not feasible to perform in batch. ${ }^{[7]}$ In this Personal Account, we highlight several of these reactions that also align with the goals outlined by the Twelve Principles of Green Chemistry. ${ }^{[1]}$ The examples described are diverse in nature and include a number of thermal, photochemical, catalytic and biphasic (e.g. gas-liquid) reactions. While they have not been scrutinized with regards to their overall greenness, our aim is simply to illustrate how our goals for improved reaction efficiency in terms atom economy, product yields and reaction rates, offer opportunities within the context of green chemistry. We also aim to demonstrate the feasibility of chemical and solvent waste reduction within a flow synthesis design as well as highlight the merits of real-time monitoring for maintaining the integrity of larger-scale production. Overall, our hope is that the reader considers the prospects of continuous flow as a stepping stone towards greener, more efficient syntheses.

Justin Lummiss received his Ph.D. in 2015 from the University of Ottawa, for work with Deryn Fogg on mechanistic organometallic chemistry in olefin metathesis. He is presently an NSERC postdoctoral fellow with Timothy Jamison at MIT.

Peter Morse studied Structural Biology and Chemistry at the University of Connecticut (B.S.). In 2010, he joined the lab of David Nicewicz as a graduate student at the University of North Carolina at Chapel Hill. His work there focused on the development of new synthetic methods in the burgeoning field of photoredox catalysis. After obtaining his Ph.D. in 2015, Peter is now a postdoctoral researcher in the lab of Tim Jamison. His current research focuses on developing new methods to synthesize bioactive molecules in flow.

Rachel Beingessner obtained her Ph.D. at the University of Ottawa in 2007 and then joined the National Institute for Nanotechnology - National Research Council Canada for one year of postdoctoral training prior to transitioning to a staff research position. In 2015, she joined the Chemistry Department at MIT where she currently works as a Research Scientist.
Tim Jamison was born in San Jose, CA, and grew up in neighboring Los Gatos, CA. He received his undergraduate education at UC Berkeley, where he conducted research in the laboratory of Prof. Henry Rapoport for nearly three years. He was then a Fulbright Scholar with Prof. Steven A. Benner at the ETH Zurich, and thereafter he undertook his Ph.D. studies at Harvard University with Prof. Stuart L. Schreiber. He then moved to the laboratory of Prof. Eric N. Jacobsen at Harvard University, where he was a Damon Runyon-Walter Winchell postdoctoral fellow. In 1999, he began his independent career at MIT, where he currently holds the positions of R. R. Taylor Professor and Head of the Chemistry Department.

\section{Flow-Enabled Greener, More Efficient Syntheses}

\subsection{Thermal Reactions - Improved Product Yields and Reaction Rates, Efficient Heat Transfer Processes}

A central pillar toward greener chemistry is the design of increasingly efficient syntheses. While reducing the volume of waste generated by reactions is the primary means for improving E-factors, additional aspects such as increasing the product yield, decreasing the reaction time, carrying out more atom economical transformations and minimizing energy loss all impact the overall efficiency of a synthesis.

One of the well-known benefits of continuous flow is the ability to safely heat a reaction mixture well beyond the boiling point, by regulating the pressure of the system with a back pressure regulator shown in Figure $\left.1 \mathrm{a} .{ }^{[\mathrm{b},}, 4 \mathrm{c}\right]$ While high temperature reactions in batch are well-established and diverse in the literature, the use of smaller reactor volumes in flow reduces the risks associated with reactor failure and also facilitates reactor containment. The latter not only has safety implications, but also enables the insulation and recapturing of lost energy more easily than in a larger batch process. ${ }^{[3 \mathrm{~d}]}$ Overall, from a manufacturing perspective, the opportunity to increase throughput and decrease residence time by accessing forcing conditions (e.g. higher temperatures and pressures) ${ }^{[8]}$ in an effective manner, has significant impacts for volume-time-output and the overall process efficiency. ${ }^{[0]}$

As an illustration of this concept, in 2010 we reported the batch and continuous flow synthesis of $\beta$-amino alcohols, a functionality found in a number of active pharmaceutical ingredients (APIs), which can be formed via the aminolysis of epoxide substrates. ${ }^{[10]}$ As shown in Figures $2 a$ and $2 b$, the ring opening of 2-(phenoxymethyl)oxirane (1) with tert-butylamine (2), resulted in complete conversion and an $82 \%$ in situ yield of amine 3 under directly comparable flow and microwave batch conditions $\left(150^{\circ} \mathrm{C}\right.$ for $\left.30 \mathrm{~min}\right)$. However, by taking advantage of the ability to safely increase the reaction temperature from 
$150{ }^{\circ} \mathrm{C}$ to $195{ }^{\circ} \mathrm{C}$ in flow, we were able to achieve an order of magnitude reduction in the time scale of the reaction ( $3 \mathrm{~min}$ vs. $30 \mathrm{~min}$ ), while maintaining a comparable yield and conversion (Figure 2b). Process intensification ${ }^{[8]}$ in flow also improved the sluggish reaction between 1,4-dihydronaphthalene oxide 4 and hindered secondary amine, indoline $\mathbf{5}$, from $39 \%$ at $150{ }^{\circ} \mathrm{C}$ to $71 \%$ at $245{ }^{\circ} \mathrm{C}$ within the same 30 min time frame (Figure 3a). Rate enhancements were likewise observed for the preparation of intermediate 9 in the synthesis of the indacaterol active pharmaceutical ingredient (API) ${ }^{[11]}$ as well as the synthesis of metaprolol 12 (Figure $3 \mathrm{~b}$ ). ${ }^{[12]}$ Both compounds were prepared in good yields with residence times of only $15 \mathrm{~min}$ and $15 \mathrm{sec}$, respectively. (a)

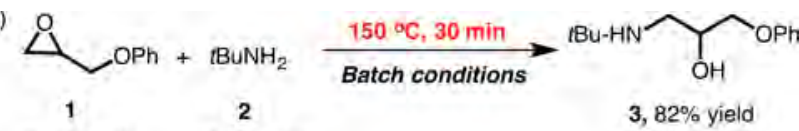

(b)

(1.0 equiv)

$$
2
$$

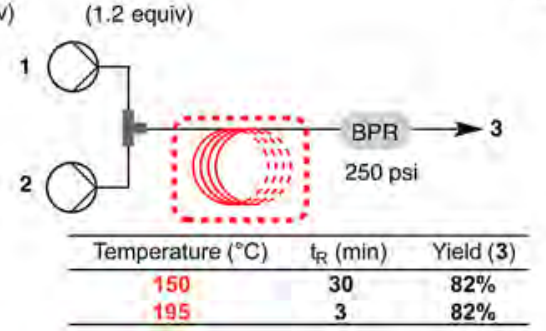

Figure 2. Reaction of 2-(phenoxymethyl)oxirane (1) with tert-butylamine (2) under (a) microwave batch conditions at $150{ }^{\circ} \mathrm{C}$ and (b) flow conditions. ${ }^{[10]}$ (a)

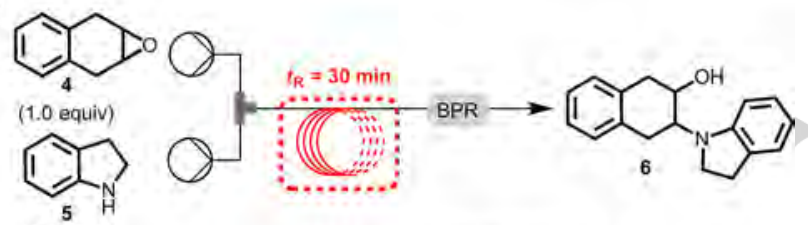

$$
\text { (1.2 equiv) }
$$

\begin{tabular}{ccc}
\hline Temperature $\left({ }^{\circ} \mathrm{C}\right)$ & BPR (psi) & Yield (6) \\
\hline 150 & 250 & $39 \%$ \\
245 & 500 & $71 \%$ \\
\hline
\end{tabular}

(b)

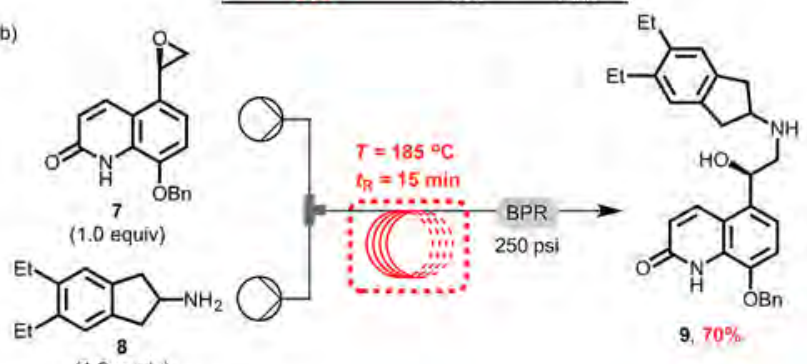

$$
\text { (1.2 equiv) }
$$

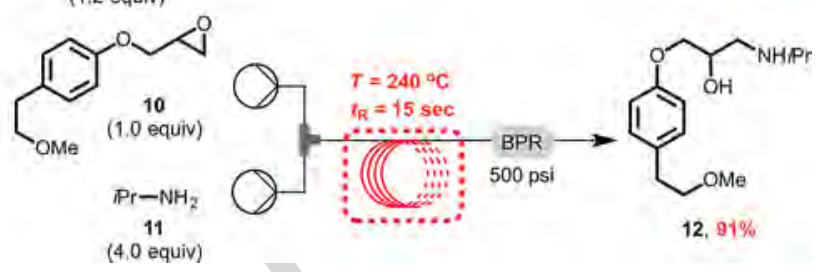

Figure 3. Process intensification in flow. Aminolysis reaction of secondary amine (4) 1,4-dihydronapthalene oxide (5). Reactions were run in $\mathrm{EtOH}$ at $1 \mathrm{M}$ concentration in epoxide (b) Aminolysis reactions applied to the synthesis of complex targets 9 and 12. The syntheses were performed in 9:1 NMP/H $\mathrm{H}_{2} \mathrm{O}$ and $\mathrm{EtOH}$, respectively. ${ }^{[10]}$
In addition to process intensification, ${ }^{[8]}$ another significant attribute of a flow reactor is the high surface area to volume ratio that results from using small diameter tubing. This enables rapid and highly controlled heat transfer processes, which in turn avoids the need for lengthy, energy-costly temperature equilibration periods typical in batch. This is particularly advantageous for multistep syntheses that require very distinct and extreme differences in temperature, such as our reported synthesis of ortho-substituted phenols, which involved the aerobic oxidation of in situ generated Grignard reagents (Figure 4). ${ }^{[13]}$ In this case, initial benzyne formation from 15 and nucleophilic addition required high temperatures $\left(80-120^{\circ} \mathrm{C}\right)$ in Reactor II, whereas the subsequent oxidation of the Grignard reagent with the renewable air feedstock in Reactor III, necessitated chilled conditions $\left(-25^{\circ} \mathrm{C}\right)$ to generate the orthosubstituted phenols $\mathbf{1 6 - 2 3}$. Rapid cooling was accomplished by simply flowing the mixture through a precooling coil for a residence time of only $1.2 \mathrm{~min}$.
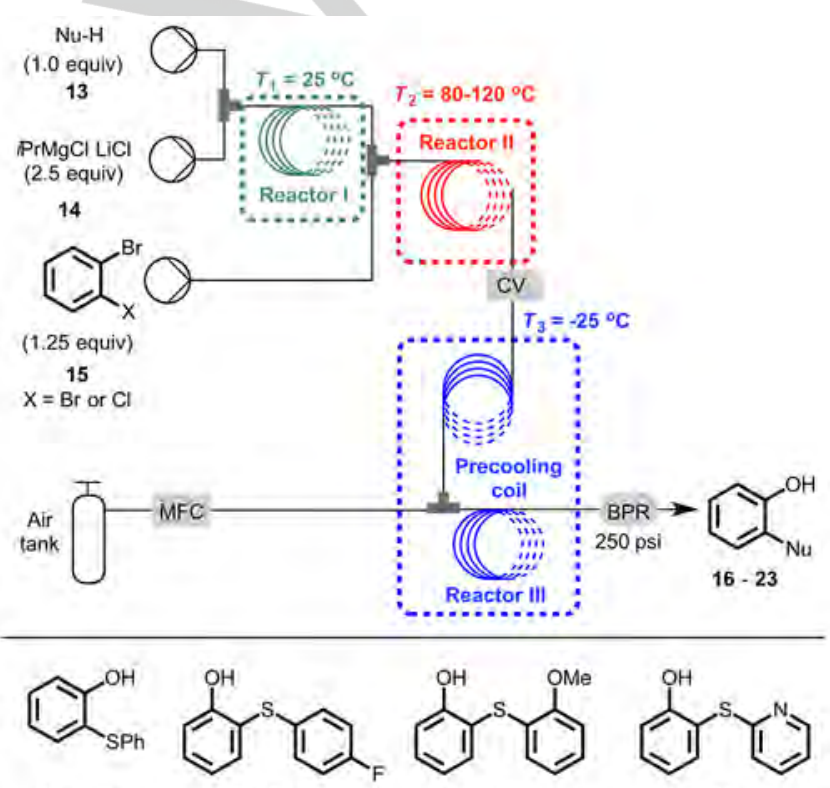<smiles>Oc1ccccc1Nc1ccccc1</smiles><smiles>[124Sn]</smiles>

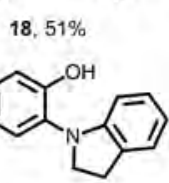

$19,46 \%$<smiles>C=CCN(c1ccccc1)c1ccccc1O</smiles><smiles>Oc1ccccc1N(Cc1ccccc1)c1ccccc1</smiles>

$20,55 \%$

$22,51 \%$

$23,33 \%$

Figure 4. Synthesis of ortho-functionalized phenols using an integrated threestep continuous flow system with three unique temperature controlled reaction zones. Total residence time for the synthesis of $\mathbf{1 6}-\mathbf{2 3}$ was 14 min. ${ }^{[13]}$

Efficient heat transfer is equally important during the quenching of a reaction that could potentially lead to an exothermic process. In this regard, the ability to continuously quench small volumes in-line or by dropwise collection of the product stream into an appropriate quenching solution, ${ }^{[14]}$ enables a controlled and convenient process, particularly where collection occurs over prolonged periods. In 2012 for example, we reported the oxidation of a variety of aldehydes and alcohols 
using bleach, a phase transfer catalyst and a $\mathrm{MeOH} / \mathrm{EtOAc}$ solvent mixture. ${ }^{[14 a]}$ In the case of 5-nitro-2-furaldehyde, a total of $11.0 \mathrm{~g}$ of methyl 5-nitro-2-furoate was generated in $75 \mathrm{~min}$, which corresponds to $211 \mathrm{~g}$ of product per day. By conducting the reaction in flow, small volumes of bleach were continuously and safely quenched at room temperature, thereby avoiding the need for more energy-intensive cryogenic temperatures. ${ }^{[15]}$

\subsection{Photochemical Reactions - Increased Efficiency and Minimization of Solvent Use}

Photochemical processes offer unique reactivity patterns and several advantages towards a more green synthesis: photons are an innocuous renewable reagent, radical initiators can be avoided, and in some cases, visible light can be sufficient to promote these reactions. ${ }^{[16]}$ In batch, however, photochemical transformations suffer from limited light penetration into the reaction mixture, which becomes increasingly problematic on scale as described by the Beer-Lambert law. ${ }^{[6 a]}$ A flow platform, alternatively, utilizes narrow diameter tubing that results in a very short path length and, as such, a significantly more efficient irradiation process. Scaling this type of reaction in flow can easily be achieved by running parallel reactors, or, if time permits, simply increasing the duration of the product collection period. While in-house reactors such as that shown in Figure $5 a$ can be readily fashioned for UV, LED, and visible light, flow photoboxes are also commercially available (Figure $5 \mathrm{~b}$ and $5 c){ }^{[17]}$
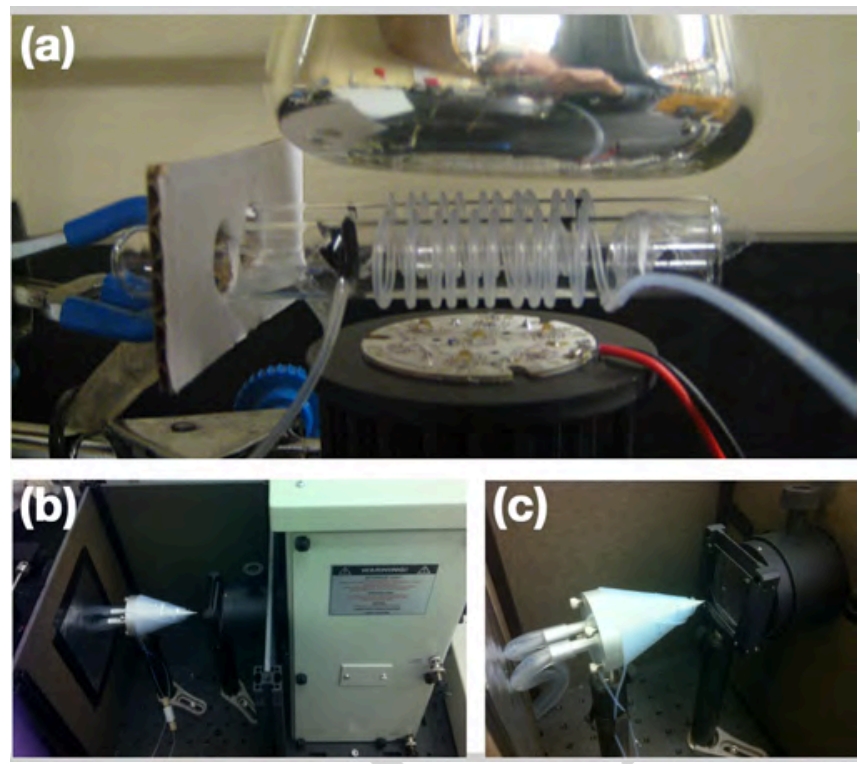

Figure 5. Representative continuous-flow photochemical reactors: (a) in house LED reactors and ( $b$ and c) commercially available UV-photobox. Image (a) is reprinted with permission from Tucker, J. W.; Zhang, Y.; Jamison, T. F.; Stephenson, C. R. J., Visible-Light Photoredox Catalysis in Flow. Angewandte Chemie, International Edition 2012, 51 (17), 4144-4147. Copyright 2012 John Wiley \& Sons.

In collaboration with Corey Stephenson's group, currently at the University of Michigan, we have translated a number of previously established batch photochemical reactions to mesoscale continuous-flow, in order to demonstrate the orders of magnitude increase in reaction efficiency that is possible within this reaction class. ${ }^{[18]}$ As a specific example, the oxidative generation of iminium ions from $\mathbf{2 4}$ was carried out using a flow reactor constructed from PFA tubing and commercial blue LEDs (Table 1). The reactive iminium 25 generated in situ was immediately trapped with a nucleophile. Under batch conditions, formation of the $\alpha$-functionalized amine $\mathbf{2 6}$ in a $95 \%$ yield from $\mathrm{N}$-aryl tetrahydroisoquinoline (24), required $1.0 \mathrm{~mol} \%$ of the $\mathrm{Ru}(\mathrm{bpy})_{3} \mathrm{Cl}_{2}$ photocatalyst and a $3 \mathrm{~h}$ reaction time. Under analogous flow conditions, a $50 \%$ reduction in catalyst loading (ca. $0.5 \mathrm{~mol} \%$ ) and a 360 -fold decrease in reaction time (ca. 0.5 $\mathrm{min}$ ) afforded the same product in a comparable $89 \%$ yield.

Table 1. Comparison of the photochemical generation and trapping of iminium ions beginning from 24 in a flow and batch platform. ${ }^{[18]}$

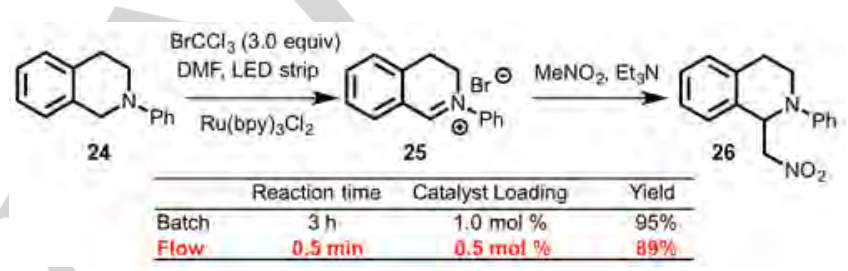

It has also been reported previously that certain photochemical reactions can be performed at significantly higher concentrations in flow reactors than comparable batch reactions, with little to no deleterious effect. ${ }^{[16 \mathrm{~d}, 18-19]}$ This improves the overall greenness of the reaction by minimizing the amount of solvent waste generated. We have similarly observed this effect in our development of a continuous flow protocol for the photochemical synthesis of the versatile, commercially-available half-sandwich catalyst $\mathrm{CpRu}(\mathrm{MeCN})_{3} \mathrm{PF}_{6}$ (28). In previously reported batch conditions, the maximum concentration attainable was only $20 \mathrm{mM}$ and the reaction required $12 \mathrm{~h}$ to reach full conversion (Figure 6). By transitioning to continuous flow, the reaction was not only accelerated from $0.5 \mathrm{~d}$ to $5 \mathrm{~min}$, but the concentration of the reaction was also increased 5 -fold, while still maintaining a near-quantitative $98 \%$ conversion. ${ }^{[20]}$ It should be noted that $100 \mathrm{mM}$ was the highest achievable concentration due to the limiting solubility of the product $\mathbf{2 8}$ in acetonitrile and not a result of a limitation in the photon flux.

Photochemical flow processes also have the potential to provide access to materials in a more atom economical manner than would typically be accomplished using more traditional synthetic strategies. ${ }^{[21]}$ As an illustration, conventional methods for amide and peptide bond formation rely on the condensation of carboxylic acids and amines and typically require the use of stoichiometric quantities of an activating agent. In contrast, we recently showed that a photochemical rearrangement of nitrones 29 in flow via oxaziridine intermediates $\mathbf{3 0}$, can provide facile access to range of amides, dipeptides, tetrapeptides, as well as symmetrical diamides in reasonable yields (Figure 7, compounds 31 - 40). ${ }^{[2]}$ For instance, complete photochemical conversion of the corresponding nitrone to amide $\mathbf{3 4}$ was 
observed within $10 \mathrm{~min}$, with no oxaziridine remaining and a $59 \%$ isolated yield of the desired amide. While the analogous batch reaction in an NMR tube did proceed with complete conversion, the oxaziridine was the major product over the desired amide $(7: 1) .{ }^{[2]}$ Thus, the enhanced irradiation efficiency of the flow reactor is key for promoting this atom-economical, photochemical transformation.

(a)
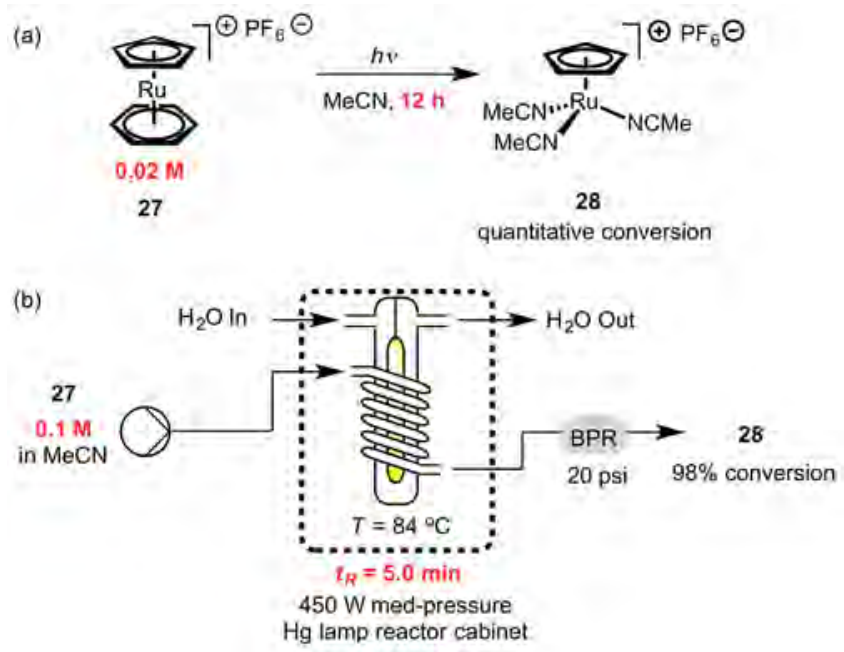

Figure 6. Photochemical synthesis of $\mathbf{2 8}$ in (a) batch and (b) continuous flow. $^{[20,23]}$

Overall, the short path length that results from the high surface area to volume ratio in mesoscale continuous flow reactors offers enormous opportunities for increased yields, decreased time-scales, and reductions in catalyst loadings within photochemical reactions. In combination with the potential use of more concentrated solutions relative to batch, photochemical flow reactions bode well towards the goals of greener chemistry.

\subsection{Catalytic Reactions - Reactor Tubing as a Catalyst Source}

The development of catalytic methods and the Principles of Green Chemistry ${ }^{[1]}$ pursue a common endeavour; both seek to develop synthetic processes that maximize the yield of desired products while minimizing inputs (i.e. energy, time, etc.) and waste generated. To this end, several groups including ours have exploited the use of metal reactor tubing, as opposed to polymer tubing, to provide a direct source of catalyst in flow reactions. In 2009 for example, Bogdan and Sach ${ }^{[24]}$ reported the synthesis of a library of 1,4-disubstituted 1,2,3-triazoles using a copper flow reactor to catalyze the "click" reaction between acetylenes and organic azides. By generating these

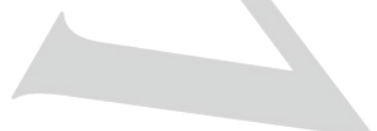

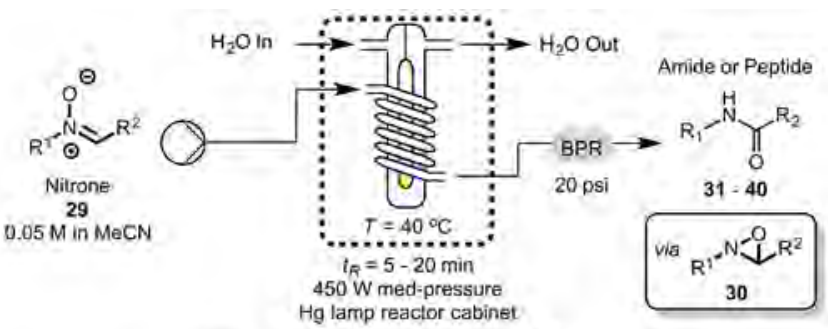

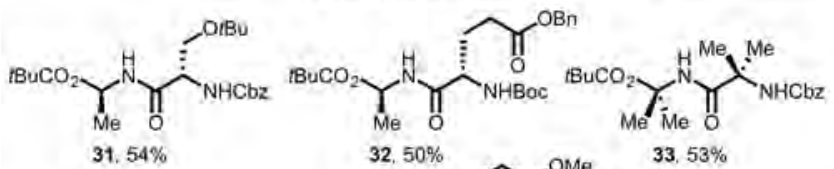

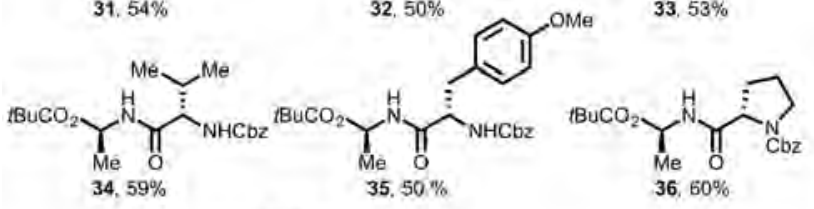

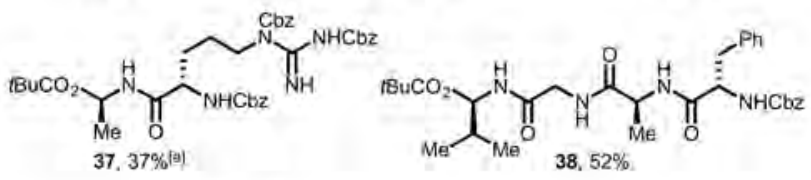<smiles>[Y19]C(NC(=O)[C@H](CCCCNC(=O)OCC)NC(=O)OCc1ccccc1)C(=O)OC(C)(C)C</smiles>

Figure 7. Examples of dipeptides, tetrapeptides, and symmetrical diamides accessed via the continuous-flow photochemical rearrangement of the corresponding nitrones. ${ }^{[22]}{ }^{[a]}$ Synthesis was performed in benzene.

azides in situ from alkyl halides and sodium azide,${ }^{[2]}$ the need for their handling was also eliminated, thereby creating a much safer and highly efficient process compared to batch protocols. Macrocyclizations using a similar strategy were also demonstrated by the same group shortly thereafter. ${ }^{[25]}$ In 2014, we reported the continuous flow synthesis of rufinamide (43), an anticonvulsant medication, that was prepared utilizing a copper flow reactor to catalyze the click reaction between an in situ formed aryl azide and propiolamide. As shown in Figure 8, the API was obtained in $92 \%$ yield using a telescoped 3-step process. ${ }^{[26]}$ This convergent synthesis compares favorably with Novartis' patented route to rufinamide, which involves isolation and handling of 2,6-difluorobenzyl azide and utilizes flammable and toxic 2-chloroacrylonitrile as a reagent. ${ }^{[27]}$

In addition to the triazole synthesis described previously, we have also illustrated the use of a copper-tubing reactor (Figure 9) for atom economical and generally high-yielding Sonogashira cross-coupling, protiodecarboxylation and Ullmann coupling reactions. ${ }^{[26,28]}$ In the case of the Sonogashira reaction (Figure 10), products $\mathbf{4 6} \mathbf{-} \mathbf{5 0}$ were generated after a $\mathbf{3 0}$ min residence time at $170^{\circ} \mathrm{C}$ in DMF and only required tetra- $n$-butylammonium acetate (TBAA) as a base. ${ }^{[28]}$ Although leached copper was observed from the reactor, it was easily removed using the solidsupported metal scavenger, Quadrapure Thiourea. 


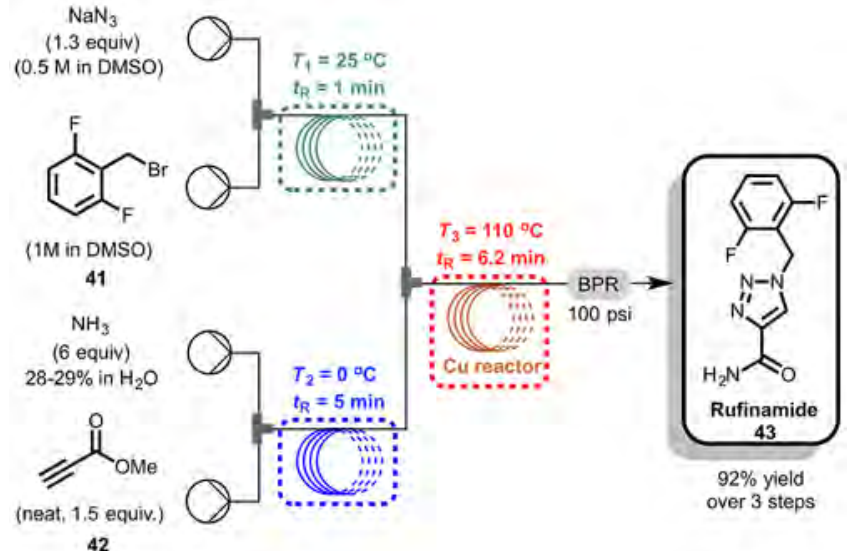

Figure 8. Copper-tube flow reactor used in the in the continuous flow synthesis of rufinamide $43 .^{[26]}$
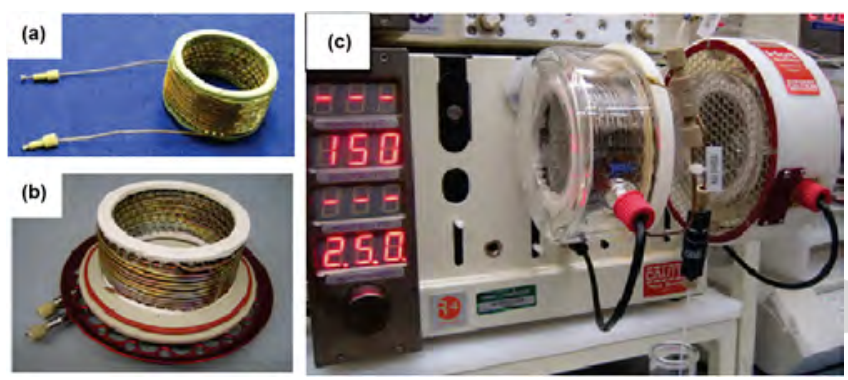

Figure 9. (a) Copper flow coil. (b) High temperature copper tube flow reactor with the top metal jacket removed (1.0 mm inner diameter tubing). (c) Heated copper tube flow reactor using a Vaportec R4 heating module. Reprinted with permission from Zhang, Y.; Jamison, T. F.; Patel, S.; Mainolfi, N., Continuous Flow Coupling and Decarboxylation Reactions Promoted by Copper Tubing Organic Letters 2011, 13 (2), 280-283. Copyright 2011 American Chemical Society.

Protiodecarboxylation reactions were also accomplished as shown in Figure 11, by simply flowing the corresponding aromatic or heteroaromatic substrates $\mathbf{5 1}$ through copper tubing at $250{ }^{\circ} \mathrm{C}$, without any additives, catalysts or ligands, to give products $\mathbf{5 2}$ - $\mathbf{5 8}$. Whereas this reaction would pose safety concerns in batch due to the gas evolution and use of high temperatures, the smaller amounts of gas continuously generated in flow and superior reaction control mitigated such safety concerns. While not implemented in this example, it is worth noting that commercially available degassing reactors utilizing DuPont's gas permeable Teflon AF, can remove gaseous by-products during flow reactions. ${ }^{[5 b]}$

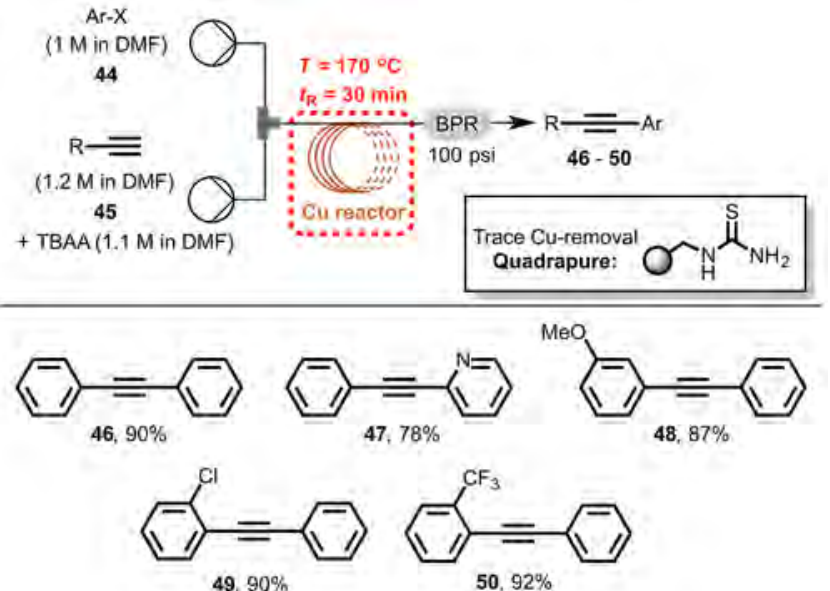

Figure 10. Sonogashira cross-coupling reaction conducted in a copper-tube flow reactor. ${ }^{[28]}$

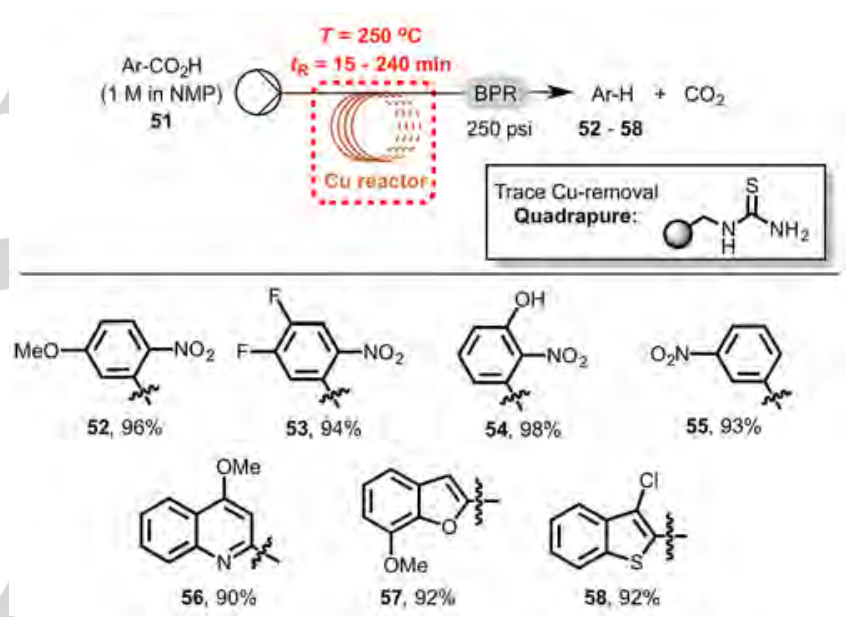

Figure 11. Protiodecarboxylation reactions conducted in a copper-tube flow reactor. $^{[28]}$

Finally, Ullmann coupling products 61 - 68 presented in Figure 12 , were produced in a copper flow reactor heated to $150{ }^{\circ} \mathrm{C}$ without the need for additional catalysts or ligands when TBAA was used as a base and acetonitrile as a solvent. Interestingly, although the conversion of 69 to 61 in flow was $65 \%$ after a residence time of $30 \mathrm{~min}$ as shown in Table 2, Entry 2 , carrying out the analogous reaction in a microwave batch reactor using $10 \mathrm{~mol} \%$ of copper powder (Entry 3 ) or the highly soluble copper iodide (Entry 4), gave only $31 \%$ and $50 \%$ conversion respectively. Although further studies are warranted, the improved efficiency of the copper-tubing catalysis relative to batch copper catalysis is a significant benefit that likely stems from the enhanced mass and heat transfer offered by the flow platform, in combination with facile formation of the active catalytic species on the surface of the reactor. 

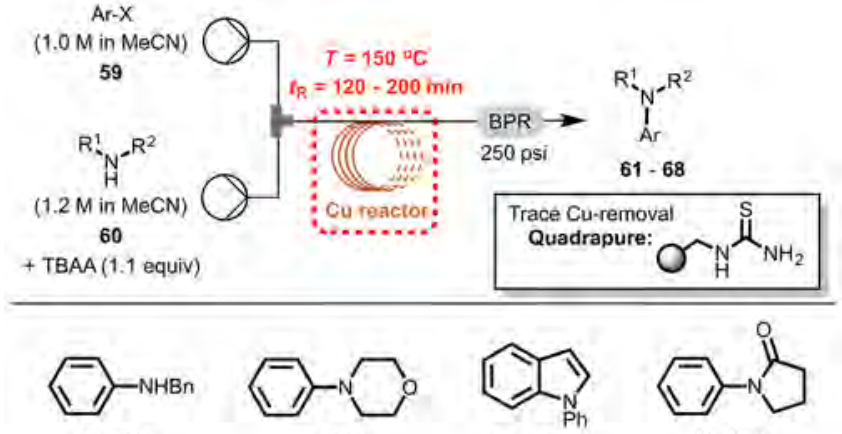

$61,74 \%$

62. $76 \%$

$63,83 \%$

64. $95 \%$
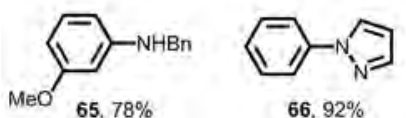

$66,92 \%$
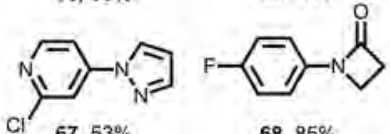

$68,85 \%$

Figure 12. Ullmann cross-coupling reactions conducted in a copper-tube flow reactor. ${ }^{[28]}$

Table 2. Comparison of batch and copper-tube flow reactors in an Ullmann coupling reaction. ${ }^{[28]}{ }^{[a]}$ Conversions are based on ${ }^{1} \mathrm{H}$ NMR analysis of the crude materials. ${ }^{[b]}$ Reaction was run in a PFA tube.

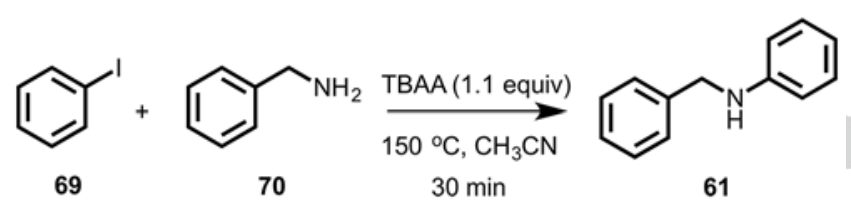

Entry Method Cu Source Cu Loading Conv. (\%)

\begin{tabular}{lllll} 
& & $(\mathrm{mol} \%)$ & \\
\hline 1 & Flow $^{[\mathrm{b}]}$ & None & 0 & 0 \\
2 & Flow & $\begin{array}{l}10 \mathrm{~mL} \text { Cu tube flow } \\
\text { reactor }\end{array}$ & Unknown & 65
\end{tabular}

3 Microwave Cu powder

$(400 \mathrm{~W})$

(Batch)

$4 \quad$ Microwave

$(400 \mathrm{~W})$

(Batch) enabling a large surface area to be exposed to the gas at any given time. Under typical flow rates used in a mesoscale reaction, friction from the sides of the reactor also create very efficient mixing, resulting in a constant supply of "gas-depleted" liquid to the liquid/gas interface. In combination with the use of higher internal pressures to aid in gas dissolution, the significantly increased interfacial contact offered in flow can dramatically improve the efficiency of gas-liquid biphasic reactions compared to traditional batch techniques. Furthermore, in situations where superstoichiometric gas is utilized, its eventual removal is straightforward using integrated vacuum degassing chambers, whereas in batch the process can be timeconsuming as well as laborious. Consequently, the utilization of renewable synthetic building blocks such as molecular oxygen or carbon dioxide gas, become more attractive reagents on the quest to achieving greener chemistry.
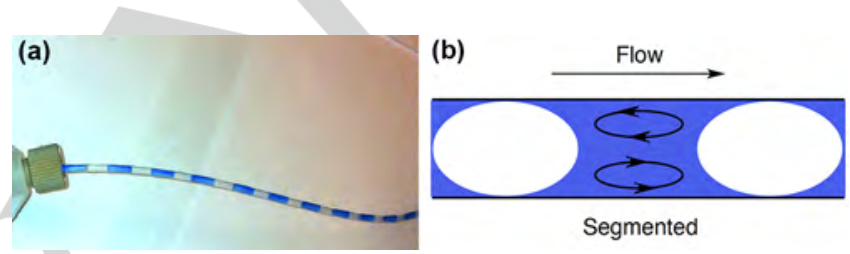

Figure 13. Biphasic gas-liquid reactions in flow (solution blue, gas colorless) (a) distribution of gas slugs during segmented flow and (b) enhanced mixing in flow during segmented flow.

Carbon dioxide is a particularly interesting renewable synthetic reagent given its potential to replace toxic $C_{1}$ building blocks such as phosgene. To that end, in 2013 we demonstrated the application of carbon dioxide in the flow synthesis of synthetically versatile cyclic carbonates. ${ }^{[29]}$ As shown in Figure 14a, electrophilic activation of epoxide starting materials 71 by in situ bromine, generated from $\mathrm{N}$-bromosuccinimide (NBS) and benzoyl peroxide (BPO), along with nucleophilic activation of carbon dioxide by DMF, enabled a range of cyclic carbonates 72 - 81 to be prepared. Although moderate yields of the products were obtained in some cases, the reaction conditions were relatively mild ( $30 \mathrm{~min}$ at $120^{\circ} \mathrm{C}$ and a pressure of only $100 \mathrm{psi}$ ) compared to other published batch protocols. For example, a recent approach utilizing a solid supported NHC to activate carbon dioxide, required more energy-intensive conditions, including significantly longer ( $24-48 \mathrm{~h}$ ) reaction times as well as higher pressures $(290 \mathrm{psi})$ to provide similar product yields (Figure 14b). ${ }^{[30]}$

Along with cyclic carbonates, the use of carbon dioxide gas for the synthesis of asymmetric ketones $86-97$ from organometallic reagents in flow has also been reported by our group as illustrated in Figure $15 .{ }^{[14 b]}$ Notably, the high interfacial contact between the gas and liquid phases enhanced the selectivity and reactivity of the carboxylation step, and resulted in nearly complete suppression of undesired symmetric ketones typically observed in batch. ${ }^{[31]}$ Controlled quenching also suppressed the formation of unwanted tertiary alcohol byproducts. Importantly, unlike typical batch protocols which use flow rates of the liquid and gas streams, the size and perio of these slugs can be modulated and controlled, thereby 
high concentrations of dry ice at low temperatures, ${ }^{[31 a, 31 b]}$ the use of a flow platform enabled greener conditions, namely room temperature and ambient pressure.
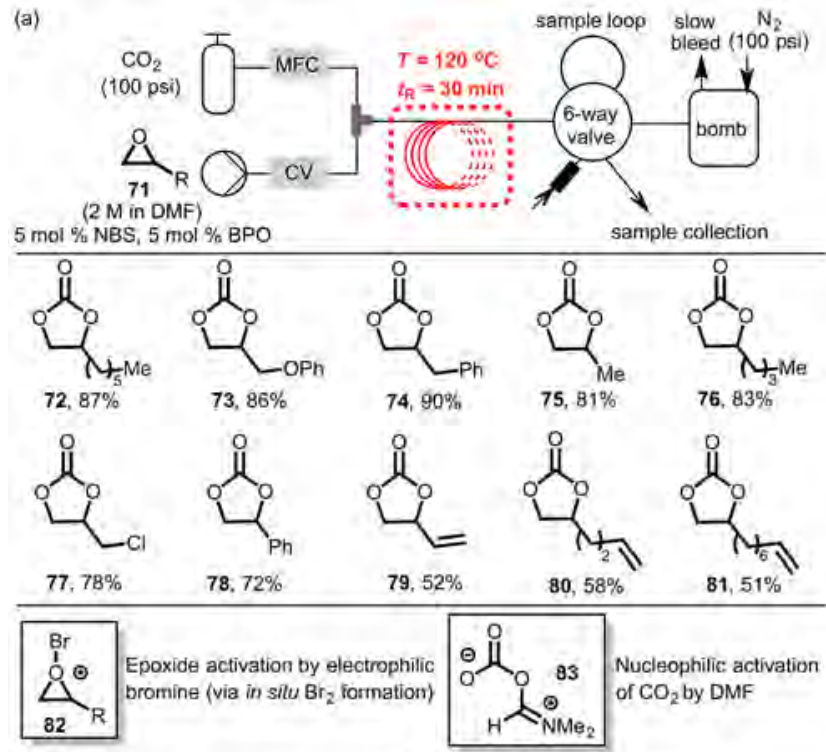

(b)

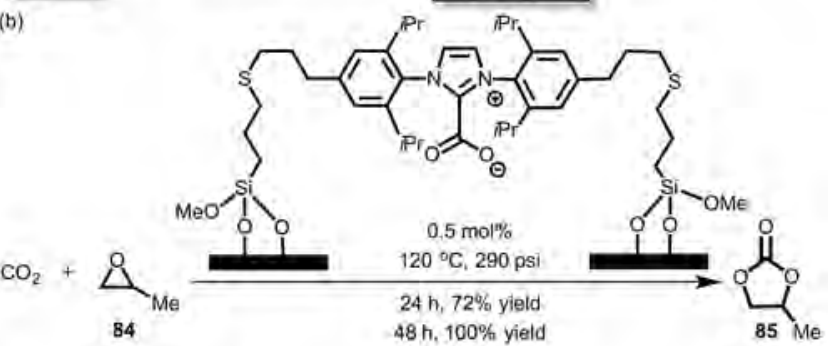

Figure 14. (a) Synthesis of cyclic carbonates from epoxides and carbon dioxide in flow. ${ }^{[29]}$ (b) Representative batch reactor synthesis of cyclic carbonates from epoxides and carbon dioxide. ${ }^{[30]}$

Overall, these two examples demonstrate the opportunity that flow provides for the facile utilization of renewable gaseous feedstocks, while also improving overall reaction efficiency. While we have also explored the use of oxygen gas in flow as briefly mentioned in Section 2.1, tremendous opportunities exist for the application of other renewal gases including hydrogen as well as carbon monoxide as demonstrated in the literature ${ }^{[32]}$ As a notable side point, liquid-gaseous reactions in batch typically require investment in specialized high pressure reactors. In a flow platform alternatively, no additional investment beyond the standard equipment (Figure 1) is necessary.

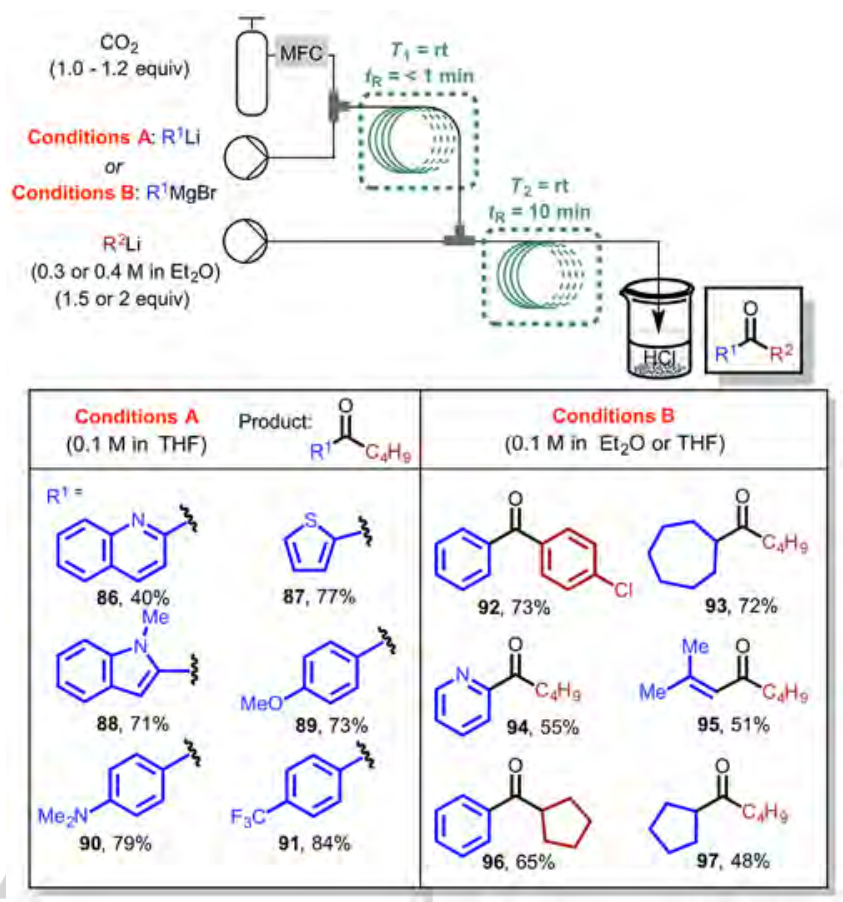

Figure 15. Synthesis of asymmetric ketones in flow. ${ }^{[14 b]}$

\subsection{Solvent Minimization for Waste Reduction}

One of the more obvious mechanisms for designing greener synthetic processes lies in the management of waste production. While improving the atom economy of a reaction is an important aspect to this, the majority of waste generated often stems from reaction and purification solvents. Thus, one strategy to minimize waste is to conduct syntheses under highly concentrated or even neat conditions. In batch however, solvents dissipate heat, ${ }^{[33]}$ which mitigates exotherm production and ensuing chemical decomposition. Fortuitously, the excellent heat transfer properties in flow described in Section 2.1, permits neat or highly concentrated reactions to be performed more safely. ${ }^{[34]}$ The ability to achieve and maintain high pressures as well as high temperatures, also implies that hot melts produced from solid reagents, starting materials or their products from a reaction, can be flowed though the system. Added to this, is the ability to telescope multistep sequences to avoid wasteful solvent need during isolation and purification steps.

A proof-of-principle demonstration of waste minimization in flow was the solvent-free and atom economical end-to-end continuous flow synthesis of the antihistamine, diphenhydramine hydrochloride recently reported by our group (Figure 16). It features a nucleophilic substitution reaction followed by real-time crystallization of the API. ${ }^{[35]}$ By combining the liquid chlorodiphenylmethane (98) and dimethylaminoethanol (99) in a flow reactor at $175{ }^{\circ} \mathrm{C}$, the resulting diphenylhydramine hydrochloride (100), which has a melting point of $168^{\circ} \mathrm{C}$, was successfully flowed in the form of an ionic liquid. Direct crystallization of the API salt was then achieved through the 
addition of a minimum amount of pre-heated $\left(175{ }^{\circ} \mathrm{C}\right.$, to avoid immediate, inline product precipitation) isopropanol anti-solvent $(1: 1, v: v)$, followed by cooling to $5^{\circ} \mathrm{C}$ in a collection tank. In this manner, $\mathbf{1 0 0}$ was provided in $84 \%$ yield as a $13.6: 1$ mixture with dimethylaminoethanol hydrochloride. Compared to Rieveschl's original batch approach ${ }^{[36]}$ that is currently used on production scale, this proof-of-principle continuous flow strategy minimizes waste by avoiding the use of a base as well as a reaction solvent. Accordingly, this work serves as an excellent example of how the merits of a flow platform can be harnessed to create a more green synthetic process.

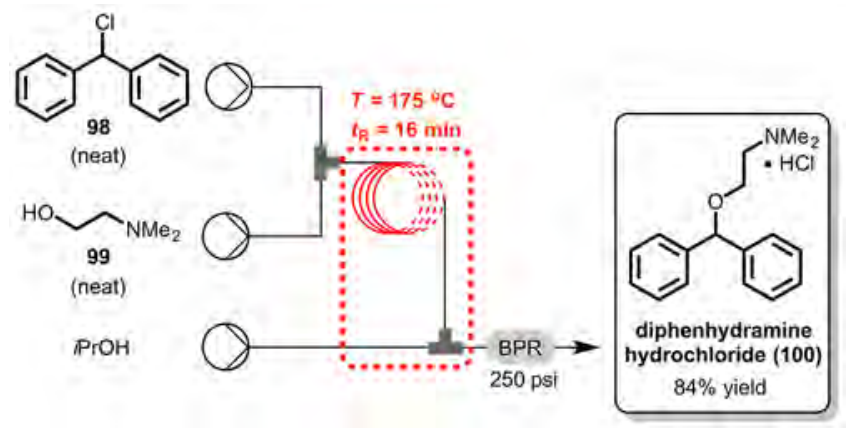

Figure 16. Flow synthesis of diphenylhydramine hydrochloride using hot melts and real-time crystallization to minimize waste production. ${ }^{[35]}$

Another well-suited illustration of the potential for waste and solvent reduction can be found in the multistep continuous flow synthesis of ibuprofen (101). ${ }^{[37]}$ As presented in Figure 17, the general synthetic process consisted of 3-bond forming steps, one work-up and one in-line liquid-liquid separation. Notably, the initial Friedel-Crafts acylation between 102 and 103 utilized neat conditions and the reagents themselves were used to solubilize the inorganic $\mathrm{AICl}_{3}$. After a residence time of only $1 \mathrm{~min}$ at $87^{\circ} \mathrm{C}$, an exothermic in-line quench of high concentrations of precipitation-prone $\mathrm{AlCl}_{3}$ was then performed using aqueous $\mathrm{HCl}$. Whereas a Syrris Asia pump was chemically compatible with the aggressive $\mathrm{AlCl}_{3}$, an HPLC pump was required to provide smooth, consistent delivery of the acidic quenching solution. Flowing through a membrane separator under high pressure (200 psi) then facilitated the separation of the aqueous byproducts $(\mathrm{Al}, \mathrm{HCl})$ from 104, again in the absence of any organic solvent.

To induce the subsequent 1,2-aryl migration step, product 104 was then treated with a solution of trimethyl orthoformate in DMF, followed by addition of neat iodine monochloride, which is commercially supplied as a liquid, solid or mixture of both depending on the polymorph composition. Since the melting point of this reagent is low (ranges from $3.9^{\circ} \mathrm{C}$ to $27.2{ }^{\circ} \mathrm{C}$ ), the need for solvent was avoided in the case of a solid, by simply heating to $35{ }^{\circ} \mathrm{C}$. A Syrris pump with a re-designed pump valve was necessary to avoid corroding by the iodine monochloride. After a residence time of only $1 \mathrm{~min}$ at $90^{\circ} \mathrm{C}$, the resulting methyl ester was then saponified and the iodine monochloride was quenched to provide ibuprofen (101) in only $3 \mathrm{~min}$ and at a production rate of $8.09 \mathrm{~g} \mathrm{~h}^{-1}$.

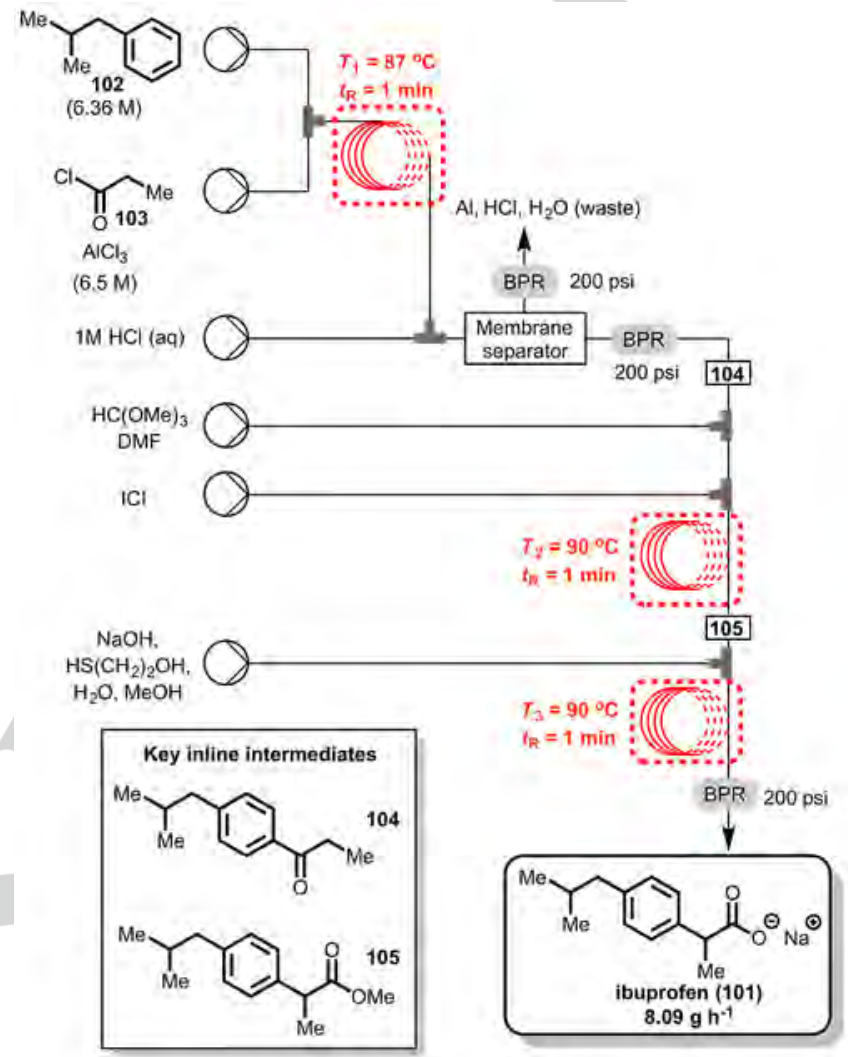

Figure 17. Continuous flow synthesis of ibuprofen features a Friedel-Crafts acylation under neat conditions, in-line separation of product 104 in its neat form and the formation of an iodine monochloride melt. ${ }^{[37]}$

\subsection{Real-time Monitoring - Maintaining Optimal Conditions and Consistent Output}

Incorporating real-time monitoring in flow chemistry enables very tight control over chemical processes that ensure maximum efficiency and minimal waste. In batch processes, there exists a homogeneous mixture of starting materials and products that in an ideal case, evolve toward complete product formation as a function of time (Figure 18, left). In a flow platform, the system contains a mixture of the reaction at all stages that progresses as a function of distance travelled through the system (Figure 18, right). The small volume of material present at any given stage of a flow process enables a very rapid response to changes in parameters, which can be used to keep the reaction within desired tolerances. More specifically, given that only a small volume of material is present at a given stage and time, any material that happens to deviate from the required tolerances, can be easily identified using real-time monitoring and diverted out of the process, thereby maintaining the integrity of the larger batch. 


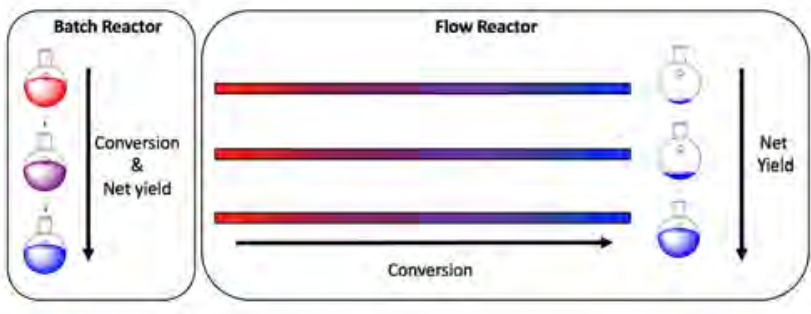

Figure 18. Simplified representation comparing reaction progress in batch reactors versus flow reactors.

Many sensors have been developed for monitoring reaction conditions in flow such as the temperature, pressure, and flow rate. By coupling sensors to appropriate software, it is possible to achieve autonomous real-time maintenance of reaction conditions. $^{[38]}$ A range of detectors have also been used for monitoring the progress of flow reactions including flow-IR, ${ }^{[39]}$ flow-UV, ${ }^{[40]}$ flow-Raman, ${ }^{[41]}$ and flow NMR. ${ }^{[42]}$ Additionally, analytic techniques such as liquid and gas chromatography ${ }^{[43]}$ and ESI mass spectroscopy ${ }^{[44]}$ are inherently flow-techniques and have also been interfaced to flow systems to provide realtime reaction monitoring (Figure 19).

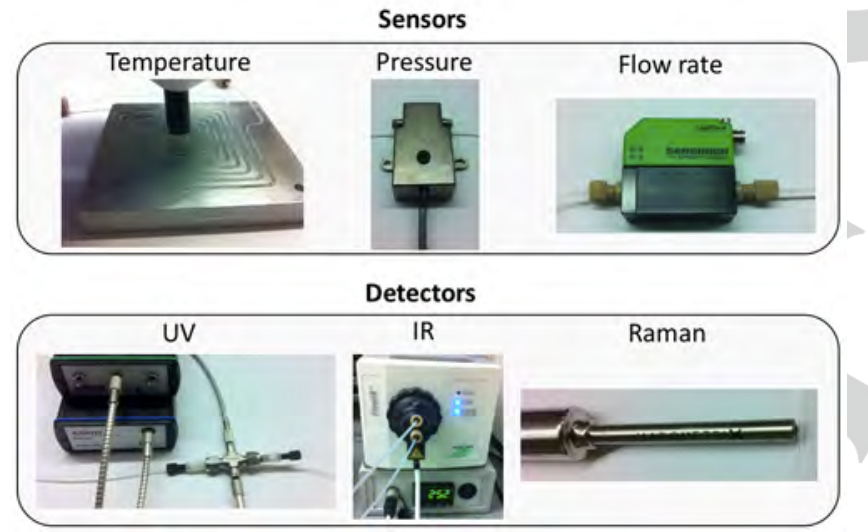

Figure 19. Representative images of common sensors and detectors that enable real-time monitoring of reactions in continuous-flow.

Our collaborative efforts in the end-to-end continuous manufacturing of pharmaceuticals that meet USP (US Pharmacopeial Convention) standards exemplifies the utility of real-time monitoring. ${ }^{[45]}$ For instance, several days into running the integrated synthesis of aliskiren hemifumarate (107), there was a disturbance in the volume maintained in the crystallization tank following the initial aminolysis reaction (Figure 20). While the volume of solution in this tank was not critical, a constant concentration of the boc-protected amine in the subsequent dilution tank was essential for maintaining the stoichiometry of the ensuing deprotection reaction with $\mathrm{HCl}$. Thus, to re-establish the desired holding volume in the crystallization tank, the system controls adjusted the flow rate of material exiting this tank.
Additionally, the flow rate of the dilution solvent (EtOAc) and the material exiting the dilution tank was adjusted in real-time to ensure constant concentration (and stoichiometry) of the bocprotected amine. By detecting and eliminating disturbances in real-time, the outcome of the integrated process (yield, purity) was maintained over extended periods with a very high degree of reproducibility.

\section{Summary and Outlook}

As illustrated herein, flow chemistry represents an enabling technology with the ability to address a number of challenges set forth by the Principles of Green Chemistry. ${ }^{[1]}$ The benefits provided in a flow platform are broad and include enhanced mixing within biphasic systems, ability to superheat reactions in a safe manner, efficient irradiation, facile integration of catalytic processes, and in-line monitoring as a means to maximize reaction product integrity. In harnessing these benefits to our advantage, we have been able to carry out numerous syntheses that have common themes of waste reduction and improved reaction efficiency (e.g. atom economy, product yields and reaction rates) relative to batch. It should be cautioned however, that assessing the overall efficiency of a particular flow process invariably requires taking many detailed factors into account that have been beyond our scope. ${ }^{[3 \mathrm{~d}, 46]}$ One noteworthy example of such a rigorous analysis on a major manufacturing process, was recently reported by Kralisch and co-workers. Their studies indicated that converting the production of epoxidized soybean oil (a plasticizer in polyvinyl chloride) from conventional batch reactors $(240,000$ tonnes/yr) to a flow process, would have a meaningful impact on lowering overall global energy demands. ${ }^{[47]}$

In terms of the equipment commercially available for various unit operations in flow, many advancements have been made in recent years, such as the development of liquid-liquid separators. ${ }^{[48]}$ Other tools such as the rotary evaporator or simple processes like product drying post-separation, however, have yet to be developed. Moreover, advances in pumping technology including chemical inertness, improvements in delivery rates and pressures, and the ability to better accommodate solid formation would be of great value. As the technology advances along these lines and the flow toolbox rounds out with increasingly financially accessible devices, so too will the scope and ease of flow processes. Accordingly, its application and synergy with the ideals of green chemistry are expected to follow suit.

\section{Acknowledgement}

We thank the Novartis-MIT Center for Continuous Manufacturing, the Bill and Melinda Gates Foundation, and the Defense Advanced Research Project Agency (DARPA) for their generous financial support over the years. J.A.M.L thanks NSERC Canada for his postdoctoral fellowship. 


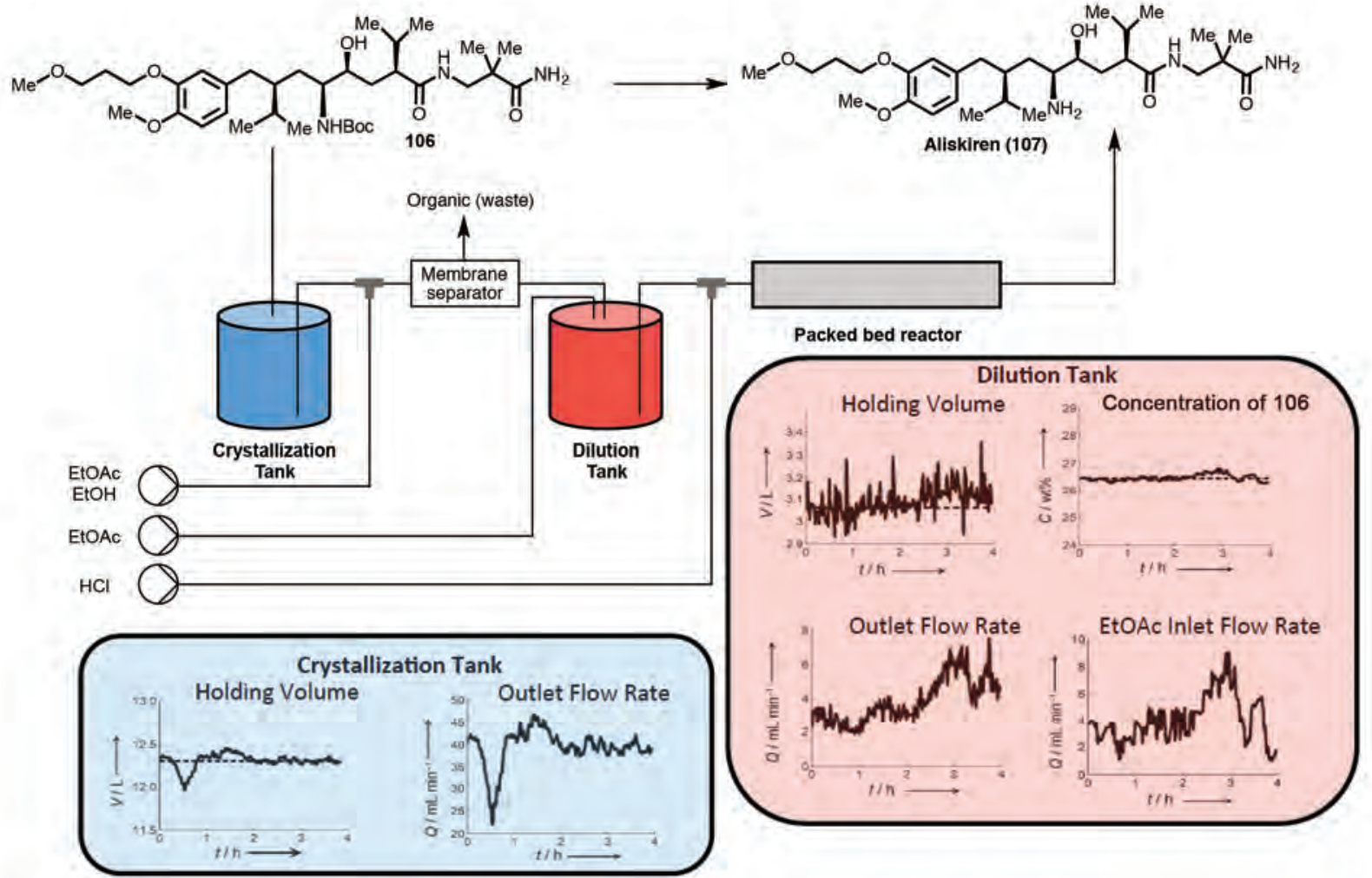

Figure 20. Disturbance mitigation in the end-to-end synthesis of aliskiren enabled by real-time monitoring. ${ }^{[45 b]}$

Keywords: Flow chemistry $\bullet$ Green chemistry $\bullet$ Real-time monitoring $\bullet$ Process intensification $\bullet$ Photochemistry

\section{References}

[1] P. Anastas, J. Warner, Green Chemistry: Theory and Practice, Oxford University Press, New York, 1998.

[2] a) R. A. Sheldon, Green Chemistry 2007, 9, 1273-1283; b) G. J. RuizMercado, A. Carvalho, H. Cabezas, ACS Sustainable Chemistry \& Engineering 2016, 4, 6208-6221; c) J. Andraos, ACS Sustainable Chemistry \& Engineering 2016, 4, 1917-1933; d) J. Auge, M.-C. Scherrmann, New Journal of Chemistry 2012, 36, 1091-1098; e) D. J. C. Constable, C. Jimenez-Gonzalez, in Handbook of Green Chemistry (Ed.: P. T. Anastas), Wiley, Hoboken, 2012, (Vol. 7) pp. 35-67; f) C. Jimenez-Gonzalez, D. J. C. Constable, C. S. Ponder, Chemical Society Reviews 2012, 41, 1485-1498; g) R. A. Sheldon, J. P. M. Sanders, Catalysis Today 2015, 239, 3-6; h) M. Tobiszewski, M. Marc, A. Galuszka, J. Namiesnik, Molecules 2015, 20, 10928-10946.

[3] a) S. V. Ley, Chemical Record 2012, 12, 378-390; b) L. Vaccaro, D. Lanari, A. Marrocchi, G. Strappaveccia, Green Chemistry 2014, 16 3680-3704; c) V. Hessel, D. Kralisch, N. Kockmann, T. Noel, Q. Wang, ChemSusChem 2013, 6, 746-789; d) S. G. Newman, K. F. Jensen, Green Chemistry 2013, 15, 1456-1472; e) S. Kobayashi, Chemistry An Asian Journal 2016, 11, 425-436; f) N. E. Leadbeater, in New and Future Developments in Catalysis: Catalysis for Remediation and Environmental Concerns (Ed. S. L. Suib), Elsevier, Waltham, 2013, pp. 19-39., 2013; g) C. Wiles, P. Watts, Green Chemistry 2012, 14, 38-54.
[4] a) F. Darvas, G. Dorman, in Flow Chemistry (Eds.: F. Darvas, G. Dorman, V. Hessel), de Gruyter, Boston, 2014, pp. 9-58. ; b) M. Fekete, T. Glasnov, in Flow Chemistry (Eds.: F. Darvas, G. Dorman, V. Hessel), de Gruyter, Boston, 2014, pp. 95-140. ; c) J. H. Bannock, S. H. Krishnadasan, M. Heeney, J. C. de Mello, Materials Horizons 2014, 1, 373-378.

[5] a) P. Plouffe, A. Macchi, D. M. Roberge, Organic Process Research \& Development 2014, 18, 1286-1294; b) M. Brzozowski, M. O'Brien, S. V. Ley, A. Polyzos, Accounts of Chemical Research 2015, 48, 349-362; c) B. J. Deadman, S. G. Collins, A. R. Maguire, Chemistry - A European Journal 2015, 21, 2298-2308; d) K. Gilmore, P. H. Seeberger, Chemical Record 2014, 14, 410-418; e) R. Porta, M. Benaglia, A. Puglisi, Organic Process Research \& Development 2016, 20, 2-25; f) J.-i. Yoshida, Y. Takahashi, A. Nagaki, Chem. Commun. 2013, 49, 9896-9904.

[6] a) Z. J. Garlets, J. D. Nguyen, C. R. J. Stephenson, Israel Journal of Chemistry 2014, 54, 351-360; b) B. D. A. Hook, W. Dohle, P. R. Hirst, M. Pickworth, M. B. Berry, K. I. Booker-Milburn, Journal of Organic Chemistry 2005, 70, 7558-7564.

[7] P. D. Morse, R. L. Beingessner, T. F. Jamison, Israel Journal of Chemistry 2016, Ahead of Print.

[8] a) T. Razzaq, C. O. Kappe, Chemistry - An Asian Journal 2010, 5, 1274-1289; b) V. Hessel, Chemical Engineering \& Technology 2009, 32, 1655-1681.

[9] a) S. Monfette, M. Eyholzer, D. M. Roberge, D. E. Fogg, Chemistry - A European Journal 2010, 16, 11720-11725; b) R. Dach, J. J. Song, F. Roschangar, W. Samstag, C. H. Senanayake, Organic Process Research \& Development 2012, 16, 1697-1706.

[10] M. W. Bedore, N. Zaborenko, K. F. Jensen, T. F. Jamison, Organic Process Research \& Development 2010, 14, 432-440. 
[11] a) B. Cuenoud, I. Bruce, R. A. Fairhurst, D. Beattie, Novartis, AG, Basel, Switzerland, W.O. Patent 0075114, 2000; b) O. Lohse, C. Vogel, Novartis, AG, Basel, Switzerland, W.O. Patent 076422, 2004; c) R. G. Sturton, A. Trifilieff, A. G. Nicholson, P. J. Barnes, Journal of Pharmacology and Experimental Therapeutics 2008, 324, 270-275.

[12] J. K. Mehra, A. Choubey, B. K. Srivastava, R. K. Porwal, P. Gautam, U.S. Patent 2005/0107635, 2005

[13] Z. He, T. F. Jamison, Angewandte Chemie, International Edition 2014 53, 3353-3357.

[14] a) A. B. Leduc, T. F. Jamison, Organic Process Research \& Development 2012, 16, 1082-1089; b) J. Wu, X. Yang, Z. He, X. Mao, T. A. Hatton, T. F. Jamison, Angewandte Chemie, International Edition 2014, 53, 8416-8420.

[15] C. Jimenez-Gonzalez, D. J. C. Constable, Green chemistry and engineering : a practical design approach, Wiley, Hoboken, 2014

[16] a) N. Hoffmann, Chem. Rev. 2008, 108, 1052-1103; b) T. Bach, J. P. Hehn, Angewandte Chemie, International Edition 2011, 50, 1000-1045 c) A. Albini, M. Fagnoni, Handbook of Synthetic Photochemistry, Wiley, Hoboken, 2010; d) E. E. Coyle, M. Oelgemoller, Photochemical \& Photobiological Sciences 2008, 7, 1313-1322; e) C. K. Prier, D. A. Rankic, D. W. C. MacMillan, Chem. Rev. 2013, 113, 5322-5363.

[17] R. Telmesani, S. H. Park, T. Lynch-Colameta, A. B. Beeler, Angewandte Chemie, International Edition 2015, 54, 11521-11525.

[18] J. W. Tucker, Y. Zhang, T. F. Jamison, C. R. J. Stephenson, Angewandte Chemie, International Edition 2012, 51, 4144-4147.

[19] a) J. P. Knowles, L. D. Elliott, K. I. Booker-Milburn, Beilstein Journal of Organic Chemistry 2012, 8, 2025-2052; b) While the extent to which a reaction can be concentrated without sacrificing yield will be limited by its intermolecular side reactions, the increased homogeneity of irradiation within flow reactors, discussed in references $16 \mathrm{~d}$ and 19a, suggests a benefit.

[20] A. C. Gutierrez, T. F. Jamison, Journal of Flow Chemistry 2011, 1, 2427.

[21] a) A. El-Faham, F. Albericio, Chem. Rev. 2011, 111, 6557-6602; b) K E. Schwieter, J. N. Johnston, Journal of the American Chemical Society 2016, 138, 14160-14169

[22] Y. Zhang, M. L. Blackman, A. B. Leduc, T. F. Jamison, Angewandte Chemie, International Edition 2013, 52, 4251-4255

[23] B. M. Trost, C. M. Older, Organometallics 2002, 21, 2544-2546.

[24] A. R. Bogdan, N. W. Sach, Advanced Synthesis \& Catalysis 2009, 351, 849-854.

[25] a) A. R. Bogdan, K. James, Chemistry - A European Journal 2010, 16 , 14506-14512; b) A. R. Bogdan, K. James, Organic Letters 2011, 13, 4060-4063.

[26] P. Zhang, M. G. Russell, T. F. Jamison, Organic Process Research \& Development 2014, 18, 1567-1570.

[27] R. Portmann, Novartis, AG, Basel, Switzerland, U.S. Patent 6,156,907, 2000 .

[28] Y. Zhang, T. F. Jamison, S. Patel, N. Mainolfi, Organic Letters 2011, 13, 280-283.

[29] J. A. Kozak, J. Wu, X. Su, F. Simeon, T. A. Hatton, T. F. Jamison, Journal of the American Chemical Society 2013, 135, 18497-18501.

[30] H. Zhou, Y.-M. Wang, W.-Z. Zhang, J.-P. Qu, X.-B. Lu, Green Chemistry 2011, 13, 644-650.

[31] a) H. Gilman, P. R. Van Ess, Journal of the American Chemical Society 1933, 55, 1258-1261; b) E. J. Soloski, C. Tamborski, Journal of Organometallic Chemistry 1978, 157, 373-377; c) J. C. Anderson, S. Broughton, Synthesis 2001, 2379-2380.

[32] C. J. Mallia, I. R. Baxendale, Organic Process Research \& Development 2016, 20, 327-360.

[33] W. Sabel, Basic Techniques of Preparative Organic Chemistry, Pergamon Press, New York, 1967.

[34] A. Weiler, Chem. Ind. 2013, 77, 20-23.

[35] D. R. Snead, T. F. Jamison, Chemical Science 2013, 4, 2822-2827.

[36] G. Rieveschl, Jr., Parke, Davis \& Co., U.S. Patent 2421714, 1947.

[37] D. R. Snead, T. F. Jamison, Angewandte Chemie, International Edition 2015, 54, 983-987.

[38] B. J. Reizman, K. F. Jensen, Accounts of Chemical Research 2016, 49, 1786-1796.
[39] a) C. F. Carter, H. Lange, S. V. Ley, I. R. Baxendale, B. Wittkamp, J. G. Goode, N. L. Gaunt, Organic Process Research \& Development 2010, 14, 393-404; b) J. S. Moore, K. F. Jensen, Organic Process Research \& Development 2012, 16, 1409-1415; c) C. F. Carter, I. R. Baxendale, M. O'Brien, J. B. J. Pavey, S. V. Ley, Organic \& Biomolecular Chemistry 2009, 7, 4594-4597.

[40] a) W. Ferstl, T. Klahn, W. Schweikert, G. Billeb, M. Schwarzer, S. Loebbecke, Chemical Engineering \& Technology 2007, 30, 370-378; b) F. Benito-Lopez, W. Verboom, M. Kakuta, J. G. E. Gardeniers, R. J. M. Egberink, E. R. Oosterbroek, A. van den Berg, D. N. Reinhoudt, Chem. Commun. 2005, 2857-2859.

[41] a) T. A. Hamlin, N. E. Leadbeater, Beilstein J Org Chem 2013, 9, 1843 1852; b) S. Mozharov, A. Nordon, D. Littlejohn, C. Wiles, P. Watts, P. Dallin, J. M. Girkin, Journal of the American Chemical Society 2011 133, 3601-3608

[42] a) V. Sans, L. Porwol, V. Dragone, L. Cronin, Chemical Science 2015, 6, 1258-1264; b) A. M. R. Hall, J. C. Chouler, A. Codina, P. T. Gierth, J. P. Lowe, U. Hintermair, Catalysis Science \& Technology 2016, 6, 84068417

[43] D. C. Fabry, E. Sugiono, M. Rueping, Reaction Chemistry \& Engineering 2016, 1, 129-133.

[44] a) S. Koster, E. Verpoorte, Lab on a Chip 2007, 7, 1394-1412; b) J. S. Mathieson, M. H. Rosnes, V. Sans, P. J. Kitson, L. Cronin, Beilstein Journal of Nanotechnology 2013, 4, 285-291.

[45] a) A. Adamo, R. L. Beingessner, M. Behnam, J. Chen, T. F. Jamison, K F. Jensen, J.-C. M. Monbaliu, A. S. Myerson, E. M. Revalor, D. R. Snead, T. Stelzer, N. Weeranoppanant, S. Y. Wong, P. Zhang, Science 2016, 352, 61-67; b) S. Mascia, P. L. Heider, H. Zhang, R. Lakerveld, B. Benyahia, P. I. Barton, R. D. Braatz, C. L. Cooney, J. M. B. Evans, T. F. Jamison, K. F. Jensen, A. S. Myerson, B. L. Trout, Angewandte Chemie, International Edition 2013, 52, 12359-12363.

[46] a) C. Jimenez-Gonzalez, P. Poechlauer, Q. B. Broxterman, B.-S. Yang, D. am Ende, J. Baird, C. Bertsch, R. E. Hannah, P. Dell'Orco, H. Noorman, S. Yee, R. Reintjens, A. Wells, V. Massonneau, J. Manley, Organic Process Research \& Development 2011, 15, 900-911; b) T. Anastas Paul, B. Zimmerman Julie, Environmental science \& technology 2003, 37, 94A-101A.

[47] D. Kralisch, I. Streckmann, D. Ott, U. Krtschil, E. Santacesaria, M. Di Serio, V. Russo, L. De Carlo, W. Linhart, E. Christian, B. Cortese, M. H. J. M. de Croon, V. Hessel, ChemSusChem 2012, 5, 300-311.

[48] a) J. G. Kralj, H. R. Sahoo, K. F. Jensen, Lab on a Chip 2007, 7, 256263; b) A. Adamo, P. L. Heider, N. Weeranoppanant, K. F. Jensen, Industrial \& Engineering Chemistry Research 2013, 52, 10802-10808; c) M. O'Brien, P. Koos, D. L. Browne, S. V. Ley, Organic \& Biomolecular Chemistry 2012, 10, 7031-7036; d) M. O'Brien, D. Cooper, Synlett 2016, 27, 164-168; e) M. O'Brien, D. A. Cooper, P. Mhembere, Tetrahedron Letters 2016, 57, 5188-5191. 


\section{Entry for the Table of Contents}

\section{PERSONAL ACCOUNT}

Intersection of Green and Flow

Chemistry. The principles of green chemistry identify the major challenges in achieving efficient, sustainable, and safe chemical transformations. Continuous flow platforms represent an enabling technology for improving reaction efficiency and reducing waste production. The intersection of these fields offers exciting possibilities for improved chemical synthesis.

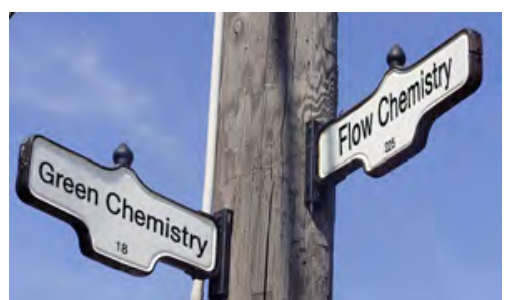

Justin A.M. Lummiss, Peter D. Morse, Rachel L. Beingessner, Timothy F. Jamison*

Page No. - Page No.

Towards More Efficient, Greener Synthesis Through Flow Chemistry 